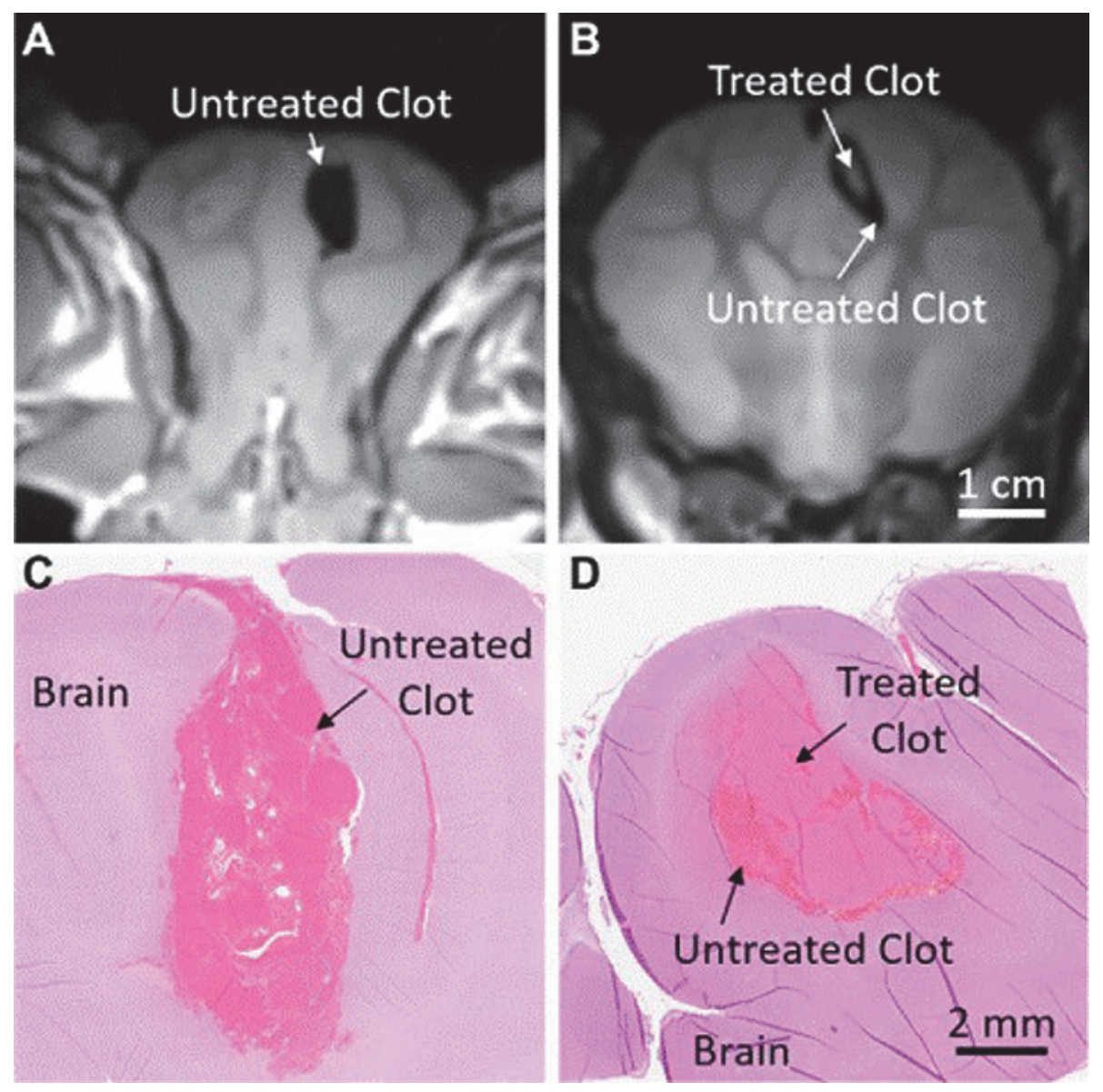

Abstract 0-006 Figure 1

Results Histotripsy was able to liquefy the center of clots without direct damage to the perihematomal brain tissue. An average volume of $0.9 \pm 0.5 \mathrm{~mL}$ was drained after histotripsy treatment. All groups showed mild ischemia and gliosis in the perihematomal region; however, there were no deaths or signs of neurological dysfunction in any groups.

Conclusion This study presents the first analysis of histotripsybased liquefaction of $\mathrm{ICH}$ in vivo. Histotripsy safely liquefies clots without significant additional damage to the perihematomal region. The liquefied content of the clot can be easily evacuated, and the undrained clot has no effect on pig survival or neurological behavior.

Disclosures T. Gerhardson: None. J. Sukovich: None. N. Chaudhary: None. T. Chenevert: None. K. Ives: None. T. Hall: None. S. Camelo-Piragua: None. B. Daou: None. Z. Xu: None. A. Pandey: None.

\section{0-007 TRENDS IN MORTALITY AND MORBIDITY AFTER TREATMENT OF UNRUPTURED INTRACRANIAL ANEURYSM IN THE UNITED STATES, 2006-2016}

${ }^{1} S$ Majidi*, ${ }^{2}$ A Mehta, ${ }^{1}$ R De Leacy, ${ }^{1} \mathrm{~J}$ Mocco, ${ }^{1} \mathrm{~J}$ Fifi. 'Neurosurgery, Mount Sinai Hospital, New York, NY; ${ }^{2}$ Neurology, Columbia University, New York, NY

\subsection{6/neurintsurg-2020-SNIS.7}

Background We aimed to assess nationally representative trends of in-hospital mortality and clinical outcome after treatment of unruptured intracranial aneurysms (UIA).
Methods The Nationwide Inpatient Sample (NIS) database from 2006 to 2016 was reviewed. Patients with unruptured intracranial aneurysm (UIA) who underwent aneurysm treatment during hospitalization were identified. Patients' demographics, comorbid conditions, length of hospital stay, rate of in-hospital mortality, discharge destination for each treatment group (microsurgical clipping and endovascular embolization). Poor clinical outcome was defined as discharge to skilled nursing facility or hospice instead of home or acute rehabilitation facility. Multivariate regression model was used to identify independent predictors of mortality and poor clinical outcome.

Results A total of 21,609 patients with UIA were identified from 2006 to 2016. The overall rate of in-hospital mortality decreased from $0.9 \%$ in 2006 to $0.2 \%$ in 2016 . Overall, $83 \%$ of the patients had favorable clinical outcome. The utilization of endovascular embolization increased from $60 \%$ in 2006 to $64 \%$ in 2016. Patients who had endovascular embolization had 3 days shorter hospital stay ( 1 vs $4, p<0.0001)$ and significantly higher rate of favorable clinical outcome compared to microsurgical clipping group $(91 \%$ vs $74 \%, \mathrm{p}<0.0001)$. Patients with age $\geq 80$ years [OR (95\% CI); p-value: 1.05 (1.02-1.11); $\mathrm{p}=0.03$ ], female gender [OR (95\% CI); p-value: 1.21 (1.07- 1.37); $\mathrm{p}=0.002]$, those with higher comorbidity index [OR (95\% CI); p-value: 1.11 (1.07- 1.18); $\mathrm{p}=0.002$ ], and patients who had microsurgical clipping [OR (95\% CI); p-value: 1.29 (1.11-1.69); p: 0.021] had higher rate of poor clinical outcome. Similarly, age $\geq 80$ years [OR (95\% CI); pvalue: 1.04 (1.01- 1.06); $\mathrm{p}=0.04]$, higher morbidity index [OR (95\% CI); p-value: $1.52 \quad(1.25-1.85) ; \mathrm{p}<0.0001]$ and 
microsurgical clipping [OR (95\% CI); p-value: 1.29 (1.111.69); $\mathrm{p}<0.0001]$ were independent predictors of mortality after treatment of the aneurysm.

Conclusions Mortality rate from treatment of unruptured intracranial aneurysm has substantially decreased in the past decade. Higher rate of morbidity and mortality is seen in patients $\geq 80$ years old, patients with multiple comorbidities and those who were treated with microsurgical clipping.

Disclosures S. Majidi: 1; C; SNIS Foundation. A. Mehta: None. R. De Leacy: None. J. Mocco: None. J. Fifi: None.

\section{0-008 ECLIPS EXPERIENCE IN WIDE-NECK BIFURCATION ANEURYSMS WITH EXTREMELY LOW DOME-TO-NECK AND ASPECT RATIOS}

${ }^{1} \mathrm{~L}$ Soerensen ${ }^{*},{ }^{2} \mathrm{~J}$ de Vries, ${ }^{3} \mathrm{M}$ Holtmannspoetter, ${ }^{4} \mathrm{~B}$ Turowski, ${ }^{5} \mathrm{~T}$ Marotta. ${ }^{1}$ Neuroradiology, Aarhus University Hospital, Aarhus N, Denmark; ${ }^{2}$ Neurochirurgisch Centrum Nijmegen, Radboud UMC, Nijmegen, Netherlands; ${ }^{3}$ Neuroradiology, Klinikum Nürnberg Süd, Nürnberg, Germany; ${ }^{4}$ Radiology, Universitetsklinikum, Düsseldorf, Düsseldorf, Germany; ${ }^{5}$ Medical Imaging, St. Michaels Hospital, Toronto, ON, Canada

\subsection{6/neurintsurg-2020-SNIS.8}

Background Bifurcation aneurysms at the basilar and carotid tip with a dome-to-neck ratio of $<1.6$ (dome width/neck width) and aspect ratio $<2$ (dome height/neck width) are challenging both to treat and also to achieve a permanent occlusion. As a consequence of the water-hammer effect, there is a tendency to coil compaction or displacement of intrasaccular devices to a degree that demands retreatment in order to avoid aneurysm rupture. Different methods and devices have been developed to overcome these challenges, and the last developed and CE approved device is the eCLIPs. eCLIPs are deployed across and outside the aneurysm neck. It diverts the blood away from the aneurysm and gives support for coils. It does not impede access to side branches nor impair flow to perforators. Here we present our experience with eCLIPs in treating basilar tip aneurysms, both as a first treatment and as adjunct to retreatment of aneurysms formerly treated with both coils and WEB. Until now around 90 eCLIPs in total have been implanted, primarily in basilar tip aneurysms and a few in carotid tip aneurysms. Of them 23 are included in this study, as they fulfill both criteria with a dome-to-neck ratio of $<1.6$ and an aspect ratio of $<1.2$. 4 patients were not available for follow up ( 2 had not reached the time for the first follow-up, one refused follow-up, and one died post procedure), so the efficacy cohort comprises of 19 patients.

Results Procedural success was achieved in 22/23 patients (96\%). There was one procedural safety event $1 / 23$ (4.3\%). No late morbidity and no recurrences were seen in the follow-up period. According to the modified Raymond-Roy score $(\mathrm{mR}-\mathrm{R})$, at the end of the index procedure 14 patients were mR-R 1 and 2, whereas 9 were $3 \mathrm{~A}$ and $3 \mathrm{~B}$. At follow-up at 11.5 months (median), 16 patients were mR-R 1,2 were $\mathrm{mR}$ R 2 and one was mR-R 3.

Conclusion Good overall procedural and follow-up results. The results are indistinguishable from that of all eCLIPs cases, including those with more favorable dome-to-neck and and aspect ratios.

Disclosures L. Soerensen: 2; C; Evasc Neurovascular Enterprises, Vancouver. J. de Vries: 2; C; Evasc Neurovascular Enterprises, Vancouver. M. Holtmannspoetter: None. B. Turowski: None. T. Marotta: 5; C; Principal, Evasc Neurovascular Enterprises, Vancouver.
$0-009$ WEB SHAPE MODIFICATION DURING FOLLOW-UP: THE BICÊTRE EXPERIENCE

J Caroff*, C Mihalea, L Ikka, A Ozanne, S Gallas, V Chalumeau, J Moret, L Spelle. Interventional Neuroradiologie, Neuri Center, Bicêtre Hospital, APHP, Le Kremlin-Bicêtre, France

\subsection{6/neurintsurg-2020-SNIS.9}

Purpose WEB device shape modification (WShM) is a potential cause of poor angiographic outcome during follow-up, despite an initial complete aneurysmal occlusion. The prevalence, the factors affecting WShM and the ways to prevent this phenomenon are only partially established.

Materials and Methods Patients treated in Bicêtre hospital with a WEB device between were included October 2015 and January 2019. Exclusion criteria were the lack of follow-up or a combined treatment. Thanks to rotational cone-beam CT (CBCT) acquisitions, systematically performed after implant and during follow-up, we have analyzed WEB morphology over time. WshM was correlated with anatomical and procedures parameters. Angiographic occlusion was evaluated according to the Bicêtre occlusion scale (BOSS).

Results 92 aneurysms were treated with a WEB device and $68 \%$ were finally included in this register. $87 \%$ of WEB devices presented WShM during follow-up (at least 20\% of height reduction). We did not find a direct relationship between the extent of WShM and occlusion rates. But in the worsening occlusion group the average WShM was significantly higher than in the stable occlusion group $(50 \%$ vs $43 \%, \mathrm{p}<0.01)$. WEB width oversizing at implant was significantly associated with a reduced WShM rate.

Conclusion CBCT allowed us to perform the first precise and quantitative analysis of WEB shape modification during follow-up. WShM is a very frequent phenomenon, negatively affecting occlusion rates. But it can be partly overcome with an appropriate oversizing strategy.

Disclosures J. Caroff: None. C. Mihalea: None. L. Ikka: None. A. Ozanne: None. S. Gallas: None. V. Chalumeau: None. J. Moret: None. L. Spelle: None.

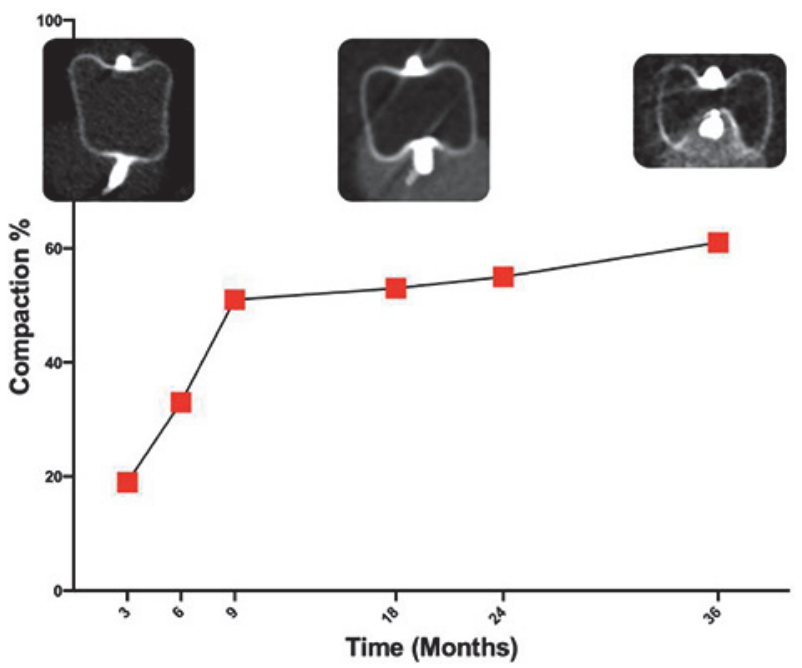

Abstract 0-009 Figure 1 Average WEB shape modification as a function of time in months. Most of the WEB reduction in height is depicted in the first 9 months then it tends to stabilize on longer follow-ups 